NASA Contractor Report 181891

ICASE Report No. 89-49

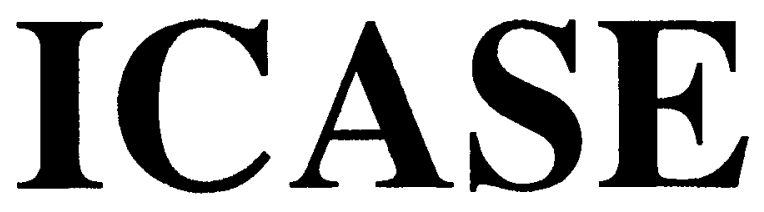

\title{
A HYBRID PERTURBATION-GALERKIN METHOD FOR DIFFERENTIAL EQUATIONS CONTAINING A PARAMETER
}

James F. Geer

Carl M. Andersen

(NASA-CR-181891) A HYBRID

N89-29122

PERTURBATION-GALERKIN METHOD FUR

OIFFERENTIAL EQUATIONS CONTAINING A

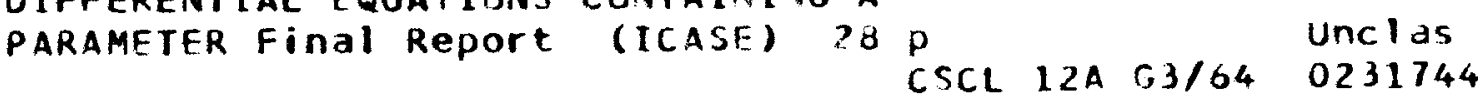

Contract No. NAS1-18605

June 1989

Institute for Computer Applications in Science and Engineering NASA Langley Research Center

Hampton, Virginia 23665-5225

Operated by the Universities Space Research Association

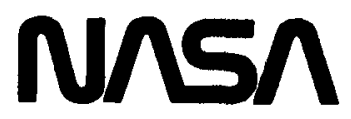

National Aeronautics and

Space Administration

Langley Research Center

Hampton, Virginia 23665-5225 


\title{
A HYBRID PERTURBATION-GALERKIN METHOD FOR DIFFERENTIAL EQUATIONS CONTAINING A PARAMETER
}

\author{
James F Geer \\ Department of Systems Science, Watson School of Engineering, Applied Science \\ and Technology, S.U.N.Y., Binghamton, NY 13901 \\ Carl M Andersen \\ Department of Mathematics, College of William and Mary, Williamsburg, VA \\ 23185
}

\begin{abstract}
A two-step hybrid perturbation-Galerkin method to solve a variety of differential equations which involve a parameter is presented and discussed. The method consists of: (1) the use of a perturbation method to determine the asymptotic expansion of the solution about one or more values of the parameter; and (2) the use of some of the perturbation coefficient functions as trial functions in the classical Bubnov-Galerkin method. This hybrid method has the potential of overcoming some of the drawbacks of the perturbation method and the Bubnov-Galerkin method when they are applied by themselves, while combining some of the good features of both. The proposed method is illustrated first with a simple linear twopoint boundary value problem and is then applied to a nonlinear two-point boundary value problem in lubrication theory. The results obtained from the hybrid method are compared with approximate solutions obtained by purely numerical methods. Some general features of the method, as well as some special tips for its implementation, are discussed. A survey of some currentresearch application areas is presented and its degree of applicability to broader problem areas is discussed.
\end{abstract}

\footnotetext{
${ }^{1}$ This research was supported by the National Aeronautics and Space Administration under NASA Contract No. NAS1-18605 while the first author was in residence at the Institute for Computer Applications in Science and Engineering (ICASE), NASA Langley Research Center, Hampton, VA 23665.
} 


\section{INTRODUCTION}

The method we wish to describe is a two-step hybrid analysis technique, which was apparently first studied by Ahmed K. Noor and collaborators in conjunction with the finite element analysis of geometrically nonlinear problems in structural mechanics (see Geer and Andersen (1989a) for several references). It is based upon the successive use of perturbation expansion methods and the classical Bubnov-Galerkin approximation technique. In the perturbation method, an approximation to the solution of a particular problem involving a parameter is developed in terms of a series of unknown functions with preassigned coefficients, i.e. gauge functions. The unknown functions are determined by solving a recursive set of differential equations which are, in general, simpler than the original governing differential equation. By contrast, in the Bubnov-Galerkin technique one seeks an approximate solution to the problem in the form of a linear combination of specified (known) coordinate functions with unknown coefficients. The coefficients are determined by demanding that the residual formed by substituting the trial solution into the governing differential equation is orthogonal to each of the coordinate functions.

The Galerkin method (see Galerkin (1915)) has, of course, been known and used for a long time. But a principle problem associated with its successful application lies in the choice of appropriate basis functions. In a series of papers Noor and his collaborators (Noor et al (1979-1988)) have shown for a variety of structural mechanics problems that the terms in a Taylor series expansion of the solution of a parameterized system of discretized equations can be particularly effective as Galerkin trial functions (or basis vectors). In addition, it has been repeatedly demonstrated that the "reduced-basis" solutions can be useful for significantly larger values of the expansion parameter than are the Taylor series solutions on which they are based. Noor and Balch (1984) and Noor, Balch and Shibut (1984) have also applied the same general principles but without discretization to some thermal analysis problems. A treatment of the reduced basis method from a mathematical point of view is given by Fink and Rheinbolt (1983).

Some general observations about the technique are the following. First, in many perturbation problems, much effort has to be expended to compute each additional term in a perturbation expansion. Through the use of the proposed hybrid method, the known perturbation terms can be exploited more fully. Secondly, another way of viewing the technique is to recognize that in many perturbation expansions the functional form of the higher-order terms can be well approximated by a linear combination of the lower order terms. Thus, much of the effect of the higher-order terms may be included by applying the reduced basis technique to the lower order terms. Finally, our investigations indicate that, while the use of a Taylor series expansion is frequently limited by a finite radius of convergence, the proposed hybrid method can sometimes yield good results well outside the radius of convergence. This is true even if that radius is zero (see Andersen and Geer (1988)).

It is our belief that the junction of perturbation and Galerkin techniques can be useful in a wide variety of application areas and in these applications the hybrid technique will give better approximations than the perturbation method alone. In this paper we will present applications of the technique independent of finite element or finite difference methods and in an area apart from structural mechanics. In particular, in the next section, we shall describe the method in more detail and then illustrate it with a simple linear two-point 
boundary value problem in section 3 . In section 4 we apply the method to a nonlinear problem in lubrication theory and use the results to determine the load carrying capacity of a slider bearing. In the final section, we make some general observations and comments on the method, as well as survey some of the current research application areas.

\section{DESCRIPTION OF THE METHOD}

The method we wish to describe is a two-step hybrid analysis technique. It is based upon the successive use of a straightforward perturbation expansion method and the classical Bubnov-Galerkin approximation technique, as outlined in the introduction. While each of these methods is useful and has been successful in providing approximate solutions to a wide variety of nonlinear (and otherwise difficult) problems, each of these techniques has certain drawbacks. The perturbation method has at least two major drawbacks. First, as the number of terms in the perturbation expansion increases, the mathematical complexity of the equations which determine the unknown functions increases rapidly. Thus, in most practical applications, the perturbation series is limited to only a few terms. A second drawback to the perturbation method is the requirement of restricting the perturbation parameter to small values in order to obtain solutions of acceptable accuracy. (These drawbacks of the perturbation method have been recognized and several modifications or extensions have been proposed, see e.g. Van Dyke (1974) and Andersen and Geer (1982).) The main shortcoming of the Bubnov-Galerkin method is the difficulty, from a practical point of view, of selecting good coordinate functions.

To illustrate the general ideas of the hybrid (or "reduced basis") method, suppose we are seeking (an approximation to) the solution $u(x)$ to the problem

$$
\mathcal{L}(u, \epsilon)=0, \quad x \text { in some domain (or interval) } \mathcal{D},
$$

where $\mathcal{L}$ is some differential operator and $\epsilon$ is a parameter. Without loss of generality, we can assume that the boundary conditions are homogeneous in $u$. We first generate the coordinate functions in a perturbation expansion of $u$ about one or more specific values of the parameter $\epsilon$, say about $\epsilon=\epsilon_{p}, p=1,2, \ldots, P$, in the form

$$
u=\sum_{k=0}^{n,-1} u_{k}^{p} \alpha_{k}^{p}(\epsilon)+O\left(\alpha_{n,}^{p}(\epsilon)\right)
$$

where $\left\{\alpha_{k}^{p}(\epsilon)\right\}$ is an appropriate asymptotic sequence of gauge functions (e.g., $\alpha_{k}^{1}=\epsilon^{k}$ for Taylor series expansions) and each $u_{k}^{p}$ can be determined completely by a standard perturbation method (e.g. a composite expansion of inner and outer expansions). A subset of all of the perturbation functions $u_{k}^{p}$ are now chosen as coordinate functions for the Bubnov-Galerkin technique and an approximation $\tilde{u}$ for $u$ is sought in the form

$$
\tilde{u}=\sum_{j=1}^{N} \delta_{j} u_{j}
$$

where the (unknown) parameters $\left\{\delta_{j}\right\}$ are functions of $\epsilon$ and represent the amplitudes of the coordinate functions $u_{j}$. Here each $u_{j}$ is one of the perturbation coordinate functions 
$u_{k}^{p}$. To determine the unknown amplitudes $\left\{\delta_{j}\right\}$, we apply the Bubnov-Galerkin technique to the governing equation (1). Thus, we substitute (3) into (1) and demand that the residual be orthogonal to the $N$ coordinate functions over the domain $\mathcal{D}$, i.e.

$$
\int_{\mathcal{D}} \mathcal{L}\left(\sum_{j=1}^{N} \delta_{j} u_{j}, \epsilon\right) u_{k} d x=0, \quad k=1,2, \ldots, N
$$

Equations (4) represent a set of $N$ equations for the $N$ unknown amplitudes $\left\{\delta_{j}\right\}$. While equations (4) must, in general, be solved numerically, solving them is simpler than numerically solving (1). In particular, for a fixed value of $\epsilon$, the solution to (4) is a point in $N$-dimensional space, where $N$ is reasonably small, while the solution of (1) is a continuous function.

We should note that this particular choice of coordinate functions overcomes the main drawback of the Bubnov-Galerkin method. By the way they are constructed, the perturbation coordinate functions are (under certain assumptions) elements of a set of functions which span the space of solutions in a neighborhood of their point of generation. Thus, they should fully characterize the solution $u$ in that neighborhood. Also, in many applications, the functions $u_{k}^{p}$ are determined by solving a set of linear equations, even though the original operator $\mathcal{L}$ may be nonlinear. The first property is necessary for the convergence of the Bubnov-Galerkin method, while the second property enhances the effectiveness of the proposed hybrid method for solving nonlinear problems.

Another important property of the proposed method is that the coordinate functions, i.e. the perturbation functions, do not need to come from a regular perturbation expansion. In fact, all that is needed is a formal asymptotic expansion of the solution to (1) for values of $\epsilon$ close to $\epsilon_{p}$ in the form of (2), where the $\left\{\alpha_{k}^{p}(\epsilon)\right\}$ are a set of appropriate gauge functions. Here we are using the term "gauge functions" (Van Dyke (1975)) in the sense that the expressions $\left\{\alpha_{k}^{p}\right\}$ form an asymptotic sequence as $\epsilon \rightarrow \epsilon_{p}$, i.e. for each fixed value

of $k$ the ratio $\alpha_{k+1}^{p} / \alpha_{k}^{p} \rightarrow 0$ as $\epsilon \rightarrow \epsilon_{p}$. Thus these expressions may involve terms like $\log \epsilon$ (Geer and Andersen (1989a)) or fractional powers of $\epsilon$ (Geer and Andersen (1989b)). Hence, it follows that the proposed method may be applied to singular, as well as regular, perturbation problems. (See Geer and Andersen (1989a). In the following section, we shall illustrate our method using the regular perturbation expansion about $\epsilon=0$ for a simple two-point boundary value problem, while in section 4 we shall apply the method using both a regular and a singular perturbation expansion of the solution.

\section{A SIMPLE EXAMPLE}

To illustrate the ideas just discussed, we consider the following simple two-point boundary value problem:

$$
\begin{gathered}
u^{\prime \prime}+\epsilon u=\epsilon, \quad 0<x<1, \\
\text { with } u(0)=0 \text { and } u(1)=0 .
\end{gathered}
$$


The exact solution to this problem for $\epsilon>0$ is

$$
\begin{gathered}
u=1-\cos (\omega x)-\left[\frac{1-\cos (\omega)}{\sin (\omega)}\right] \sin (\omega x), \\
\omega=\sqrt{\epsilon}
\end{gathered}
$$

This function, which is graphed iń Fig. 1, has (real) singularities at $\epsilon=\pi^{2},(3 \pi)^{2},(5 \pi)^{2}, \ldots$. A regular perturbation expansion of $u$ yields a series of the form

$$
u=\sum_{j=1}^{N} u_{j}(x) \epsilon^{j}+O\left(\epsilon^{N+1}\right)
$$

where each $u_{j}$ is a polynomial in $x$ of degree $2 j$ which vanishes at $x=0$ and $x=1$. Following the ideas discussed above, we look for an approximate solution $\tilde{u}$ in the form

$$
\tilde{u}=\sum_{j=1}^{N} \delta_{j} u_{j}
$$

where the amplitudes $\left\{\delta_{j}\right\}$ are to be determined for each value of $\epsilon$ of interest. To determine them, we substitute (8) into (5) and apply the Bubnov-Galerkin criterion (4) to obtain the set of equations

$$
\sum_{j=1}^{N} a_{k, j}(\epsilon) \delta_{j}=b_{k}(\epsilon), \quad k=1,2, \ldots, N
$$

where

$$
\begin{gathered}
a_{k, j}(\epsilon)=\int_{0}^{1} u_{k}(x)\left[u_{j}^{\prime \prime}(x)+\epsilon u_{j}(x)\right] d x, \\
b_{k}(\epsilon)=\epsilon \int_{0}^{1} u_{k}(x) d x .
\end{gathered}
$$

In this case the system of equations for the $\left\{\delta_{j}\right\}$ is linear since the original problem is linear. For example, setting $N=2$ in (9), we find

$$
\begin{aligned}
& \delta_{1}=\frac{1008 \epsilon-112 \epsilon^{2}}{1008-112 \epsilon+\epsilon^{2}}, \\
& \delta_{2}=\frac{1008 \epsilon^{2}}{1008-112 \epsilon+\epsilon^{2}} .
\end{aligned}
$$

For small values of $\epsilon$, we see that these expressions reduce to $\epsilon$ and $\epsilon^{2}$, respectively, as they should. Also, they remain finite as $\epsilon$ becomes large, which suggests that our approximate hybrid solution might be valid for larger values of $\epsilon$ than the perturbation solution on which it is based. For $N=4$, we find by using the symbolic computation system Mathematica (Wolfram (1988)) that 


$$
\begin{gathered}
\delta_{1}=-1232 \epsilon\left(-159120+18720 \epsilon-273 \epsilon^{2}+\epsilon^{3}\right) / D, \\
\delta_{2}=48048 \epsilon^{2}\left(4080-480 \epsilon+7 \epsilon^{2}\right) / D, \\
\delta_{3}=-11531520 \epsilon^{3}(-17+2 \epsilon) / D, \\
\delta_{4}=196035840 \epsilon^{4} / D, \\
D=196035840-23063040 \epsilon+336336 \epsilon^{2}-1232 \epsilon^{3}+\epsilon^{4} .
\end{gathered}
$$

For small values of $\epsilon$ we see that $\delta_{j}$ reduces to $\epsilon^{j}+O\left(\epsilon^{5}\right)$.

In Figs. 2 through 5 we compare two- and four-term hybrid solutions (8) with twoand four-term perturbation solutions (7) and with the exact solution (6). Fig. 2 shows comparisons for $\epsilon=2,4$ and 6 . This figure indicates that the two-term perturbation solution $(N=2)$ does well for $\epsilon$ up to about $\epsilon=2$, but that by $\epsilon=6$ even the four-term $\epsilon=2$, but that by $\epsilon=6$ even the four-term perturbation solution $(\mathrm{N}=4)$ has serious errors. In this figure the two-term hybrid solutions agree very well with the exact solutions.

Fig. 3 shows similar comparisons for $\epsilon$ near the radius of convergence, $\pi^{2}$, of the perturbation expansion. The two- and four-term perturbation solutions are not sensitive to the small variations in $\epsilon$ and are poor approximations. The two-term hybrid solution is still very accurate. However, for values of $\epsilon$ much closer to $\pi^{2}$ than the values used in Fig. 3, the relative error of the hybrid solution becomes large because the two-term hybrid solution predicts the singularity to be at $\epsilon=(3.141616)^{2}$ rather than at $\pi^{2}$. Higher-order hybrid solutions become more accurate in this respect. The four-term hybrid solution gives the first singularity accurate to 13 digits.

Fig. 4 compares the two- and four-term hybrid solutions with the exact solution at $\epsilon=(2 \pi)^{2}$. The two-term hybrid solution is a good approximation, but the four-term hybrid solution is much better. For values of $\epsilon$ this large the perturbation solutions are useless. Finally, in Fig. 5 we compare for $\epsilon=1.02(3 \pi)^{2}$ the two- and four-term hybrid solutions with the exact solution. The two-term hybrid solution is no longer useful, but the four-term hybrid solution is still satisfactory. In general, the larger $\epsilon$ becomes the more terms are needed for the hybrid solution to give a useful approximation.

Some of the reasons why the hybrid method provides a good approximation in this case, as well as insights this example provides for the validity of the method, will be discussed in section 5. Applications of the hybrid method to some general classes of two-point boundary value problems have been discussed in Geer and Andersen (1989b).

\section{APPLiCATION - A SLIDER BEARING PROBLEM}

We now illustrate the hybrid method for a nonlinear example. We consider the problem of determining the excess pressure $u(x)$ under a slider bearing, discussed for large bearing numbers by DiPrima (1969). Here $u(x)$ satisfies

$$
\begin{gathered}
\epsilon \frac{d}{d x}\left\{h^{3}(x)[1+u(x)] \frac{d u}{d x}\right\}=\frac{d}{d x}\{h(x)[1+u(x)]\}, \\
0<x<1, \text { with } u(0)=0=u(1) .
\end{gathered}
$$

Here $\epsilon$, the inverse of the bearing number, is defined by $\epsilon=p_{a} d^{2} /(6 \mu B U)$, where $p_{a}$ is the ambient pressure in the fluid, $d$ is the clearance of the trailing edge of the bearing, $\mu$ is 
the dynamic viscosity of the fluid, $B$ is the length of the bearing in the flow direction, and $U$ is the velocity of the bearing. The function $h(x)$ is the prescribed film thickness under the bearing normalized such that $h(1)=1$.

For small values of $\epsilon$ (large bearing numbers), DiPrima constructed an asymptotic expansion of the form (2) with $\epsilon_{1}=0$ and $\alpha_{j}^{1}=\epsilon^{j}, j=0,1, \ldots$, i.e.

$$
u=u_{0}^{1}+u_{1}^{1} \epsilon+u_{2}^{1} \epsilon^{2}+\ldots
$$

with

$$
\begin{aligned}
& u_{0}^{1}=\frac{h(0)}{h(x)}+Q\left(\frac{1-x}{\epsilon}\right)-h(0) \\
& \quad-(1-x)\left\{Q\left(\frac{1}{\epsilon}\right)-h(0)+1\right\}, \\
& h(0) \log \left|\frac{h(0)-1-Q}{h(0)-1}\right|+Q=-\frac{1-x}{\epsilon} .
\end{aligned}
$$

For large values of $\epsilon, u$ has a regular perturbation expansion of the form (2) with $\alpha_{j}^{2}=\epsilon^{-j-1}$, i.e.

$$
u=u_{0}^{2} \epsilon^{-1}+u_{1}^{2} \epsilon^{-2}+u_{2}^{2} \epsilon^{-3}+\ldots
$$

Substituting (16) into (13) and equating coefficients of like powers of $\epsilon^{-1}$ on each side of the resulting equation, we find that each $u_{k}^{2}$ satisfies a simple second-order differential equation with homogeneous boundary conditions. In particular, we find that $u_{0}^{2}$ satisfies

$$
\frac{d}{d x}\left\{h^{3}(x) \frac{d}{d x} u_{0}^{2}\right\}=\frac{d}{d x} h(x), \quad u_{0}^{2}(0)=0=u_{0}^{2}(1)
$$

whose solution is

$$
\begin{gathered}
u_{0}^{2}=\int_{0}^{x} h^{-2}(s) d s+c \int_{0}^{x} h^{-3}(s) d s \\
c=-\int_{0}^{1} h^{-2}(s) d s / \int_{0}^{1} h^{-3}(s) d s
\end{gathered}
$$

We now select one term from the small- $\epsilon$ expansion (14) of $u$ and one term from the large- $\epsilon$ expansion (16) and construct a hybrid solution $\tilde{u}$ in the form (3) with $N=2$, i.e.

$$
\tilde{u}=\delta_{1} u_{0}^{1}(x, \epsilon)+\delta_{2} u_{0}^{2}(x) \text {. }
$$

The quadratically nonlinear equations (4) for the amplitudes $\left\{\delta_{j}\right\}$ become

$$
\begin{gathered}
\int_{0}^{1}\left\{\epsilon \frac{d}{d x}\left[h^{3}[1+\tilde{u}] \frac{d \tilde{u}}{d x}\right]-\frac{d}{d x}\{h[1+\tilde{u}]\}\right\} u_{0}^{1}(x, \epsilon) d x=0 \\
\int_{0}^{1}\left\{\epsilon \frac{d}{d x}\left[h^{3}[1+\tilde{u}] \frac{d \tilde{u}}{d x}\right]-\frac{d}{d x}\{h[1+\tilde{u}]\}\right\} u_{0}^{2}(x) d x=0
\end{gathered}
$$

These equations can be solved easily (and efficiently) by using Newton's method, starting at small values of $\epsilon$ and then proceeding to larger values of $\epsilon$. 
To illustrate some results obtained by this approach we consider first a wedge-shaped slider bearing with $h(x)=2-x$. [For each of the figures in this section hybrid results are indicated by solid lines, small- $\epsilon$ perturbation expansions by lines with short dashes, large- $\epsilon$ perturbation expansions by lines with long dashes, and the numerical results (which were obtained by a shooting method) with circles.] In Fig. 6, we compare for $\epsilon=0.7$ the hybrid solution for $N=2$ with the one-term small-parameter solution (14), the one-term large-parameter solution (16) and the solution obtained by a numerical shooting method. The value 0.7 was chosen for $\epsilon$ because for the given function $h(x)$ of all positive values of $\epsilon$ this represents nearly the poorest agreement, an $L_{2}$ relative error norm of about $1 \%$, between the hybrid solution and the numerical solution. A comparison of the hybrid and numerical solutions for four other values of $\epsilon$ is shown in Fig. 7. In Fig. 8, we have plotted the amplitudes $\delta_{1}$ and $\delta_{2}$ (the coefficients of the small- and large- $\epsilon$ coordinate functions, respectively) as functions of $\epsilon$. As the figure illustrates, $\delta_{1}$ approaches 1 and $\delta_{2}$ approaches 0 as $\epsilon$ approaches zero, while $\delta_{2}$ dominates $\delta_{1}$ as $\epsilon$ approaches infinity. In Figs. 9 and 10 we have made comparisons similar to that shown in Fig. 6 , this time for film thicknesses corresponding to a parabolic shape with $h(x)=1+2(1-x)^{2}$, a case discussed by DiPrima, and to a cubic shape with $h(x)=1+(1-x)^{2}(1+2 x)$. Again, the agreement between the hybrid and numerical solutions is very good, even for the "nearly worst case" values of $\epsilon$ depicted in the figures.

As an application of these results, we compute the nondimensionalized load carrying capacity $W$ of the bearing, defined by $W=\widehat{W} /\left(p_{a} B\right)$, where $\widehat{W}$ is the load carrying capacity per unit width. $W$ is given by

$$
W=\int_{0}^{1} u(x) d x .
$$

Inserting our two-term hybrid approximation for $u$ into (21), our approximation $\tilde{W}$ to $W$ becomes

$$
\tilde{W}=\delta_{1} W_{1}+\delta_{2} W_{2}
$$

where

$$
\begin{aligned}
& W_{1}=\int_{0}^{1} u_{0}^{1}(x, \epsilon) d x \\
& =\frac{1}{2}(h(0)-3)-\frac{1}{2} Q\left(\frac{1}{\epsilon}\right)\left[1+2 \epsilon+\epsilon Q\left(\frac{1}{\epsilon}\right)\right] \\
& +\int_{0}^{1} \frac{h(0)}{h(x)} d x \\
& W_{2}=\int_{0}^{1} u_{0}^{2}(x) d x \\
& =-\int_{0}^{1} x\left[h^{-2}(x)+c h^{-3}(x)\right] d x,
\end{aligned}
$$


and $u_{0}^{1}(x, \epsilon)$ and $u_{0}^{2}(x)$ are defined by (15) and (18), respectively. In obtaining (23) we have used some of the ideas of DiPrima to evaluate explicitly some of the integrals which appear in this expression.

To compute $\tilde{W}$ from formulas (22) - (24) for a specific bearing configuration, the prescription is:

1) specify the film thickness profile $h(x)$ and the bearing number $\epsilon$;

2) calculate the quantity $Q(1 / \epsilon)$ from $(15 \mathrm{~b})$ with $x=0$;

3) evaluate the integrals involving $h(x)$ in (23) and (24) and use these results together with $Q(1 / \epsilon)$ to find the values of $W_{1}$ and $W_{2}$;

4) calculate the values of $\delta_{1}$ and $\delta_{2}$ from equations (20); and

5 ) insert the values of $\delta_{1}, \delta_{2}, W_{1}$, and $W_{2}$ into (22) to calculate $\tilde{W}$.

For the special case of a wedge slider bearing, where $h(x)=1+\alpha(1-x)$ with $\alpha>0$, these equations become

$$
\begin{gathered}
W_{1}=\frac{1}{2}(\alpha-2)+\frac{1+\alpha}{\alpha} \log (1+\alpha) \\
-\frac{1}{2} Q\left(\frac{1}{\epsilon}\right)\left\{1+2 \epsilon+\epsilon Q\left(\frac{1}{\epsilon}\right)\right\}, \\
W_{2}=\frac{1}{\alpha^{2}} \log (1+\alpha)-\frac{2}{\alpha(2+\alpha)} .
\end{gathered}
$$

Here $Q(1 / \epsilon)=Q$ is determined from equation (15b) with $x=0$, which thus becomes

$$
(1+\alpha) \log \left|\frac{\alpha-Q}{\alpha}\right|+Q=-\frac{1}{\epsilon} .
$$

In Fig. 11 we have plotted the load carrying capacity, $W$, for the wedge-shaped bearing with $h(x)=2-x$ (i.e., $\alpha=1$ ) as $\epsilon$ varies between 0 and 0.6 . Shown are the twoterm hybrid approximation, $\tilde{W}$; the one-term small- $\epsilon$ approximation, $W_{1}$ (see Diprima (1969)); the one-term large- $\epsilon$ approximation, which is $W_{2} / \epsilon$; and a few points calculated numerically. As expected, the hybrid and the small- $\epsilon$ perturbation calculations agree near $\epsilon=0$. Also the hybrid and the large- $\epsilon$ perturbation calculations agree for very large $\epsilon$.

the large- $\epsilon$ perturbation calculations agree for large $\epsilon$. While there is an intermediate range of values of $\epsilon$ for which the small- $\epsilon$ and large- $\epsilon$ perturbation results agree very well, neither perturbation result has much accuracy there. On the other hand, it appears that the hybrid and numerical results are in very close agreement with each other throughout the entire range $0<\epsilon<\infty$.

\section{DISCUSSION}

We believe that the general technique we have outlined here provides a very useful method for analytically analyzing the behavior of solutions to differential equations over much wider ranges of parameter values than can be obtained by the perturbation or Galerkin methods alone. The key seems to be that the perturbation functions are particularly 
effective as "trial" functions for the Galerkin method, since they characterize the solution well for certain "extreme" values of the parameter.

Some observations about the hybrid method, as we have described it, are the following. When higher-order terms in a perturbation expansion of the solution can be well approximated by linear combinations of lower order terms, the hybrid method appears to be an effective way to include some of the effects of these higher-order terms without actually computing them. In a practical problem, this property of the higher-order terms may manifest itself in the following way. A sequence of perturbation approximations based on lower order terms may appear to have approximately the right "shape" for the solution, but not the correct amplitude. This property is illustrated in our simple example in section 3 for small values of $\epsilon$ by comparing the perturbation approximations based on two and four terms. In this case, the hybrid approximation based on just the first two perturbation coefficient functions provides the necessary corrections for the "amplitudes" of these terms and results in a greatly improved approximate solution. More formally, this property can be expressed by the degree of linear dependence among the perturbation coordinate functions. If the perturbation functions are highly dependent, then only a few of them are needed in the hybrid solution to provide a reasonable approximation to the actual solution. An extreme case of this was discussed by Geer and Andersen (1989a) in connection with a problem in slender body theory. In this case all of the perturbation coordinate functions are multiples of the first coordinate function and hence all of the functions are dependent. While the partial sums in the perturbation solution converge (slowly) to the exact solution, the hybrid solution based on only one perturbation coordinate function reproduces the exact solution to the problem.

In a particular problem, the degree of dependence of a set of perturbation coordinate functions on the domain of interest may have to be obtained empirically. For example, the perturbation coordiante functions associated with our simple example in section 3 are polynomials of different orders and hence are definitely linearly independent. However, on the interval $[0,1]$ they have a very high degree of linear dependency, which can be illustrated by normalizing the first several coordinate functions and plotting them on the same graph. (We have plotted the first fifteen (normalized) polynomials associated with our simple example and have found that they all have essentially the same parabolic shape, with only two or three curves being distinguishable at usual plotting resolution.) As a practical matter, a set of perturbation coordinate functions that are highly dependent may cause some numerical sensitivities when solving equations (4) for the amplitudes $\delta_{j}$. Intuitively, this follows from the form of equations (4), which differ from each other only by the choice of the "test" functions $u_{k}$ which multiply the residual in the integrand. In this case, we have found it helpful to apply an orthonormalization process (such as the Gram-Schmidt process) to the the set $\left\{u_{k}\right\}$ and use the resulting set of orthonormal functions as the basis for the hybrid method. These steps will lead to the same hybrid approximation as would be obtained without the orthonormalization process, but the equations (4) will be better conditioned.

It may be observed that from the Galerkin point of view the perturbation coordinate functions (7) generated for the simple two-point boundary-value problem are not very exciting. For example, the first two such functions are 


$$
\begin{aligned}
& u_{1}=-x(1-x) / 2, \\
& u_{2}=-x(1-x)\left(x^{2}-x-1\right) / 24 .
\end{aligned}
$$

The factors $x$ and $1-x$ are needed to satisfy the boundary conditions; the problem has built-in symmetry about $x=1 / 2$; and the overall numerical factors are immaterial. It turns out that the basis for the space spanned by the first $N$ functions $u_{j}$ is simply the sequence $\left\{x(1-x)(x-1 / 2)^{2(j-1)}\right\}$ with $j=1,2, \ldots, N$, and this set of trial functions could easily have been conjectured for use in a stand-alone Galerkin solution. Nevertheless, it still may be said that the perturbation coordinate functions are good trial functions, that a very significant improvement over the perturbation result has been demonstrated, and that this improvement can be expected even for problems for which no "obvious" set of trial functions can be conjectured.

In the simple example of section 3, we illustrated the fact that the hybrid method may produce meaningful approximations to the actual solution even well beyond the radius of convergence of the perturbation series. This is also illustrated by the simple example discussed by Geer and Andersen (1989a), as well as by the eigenvalue problem discussed by Andersen and Geer (1988). In the eigenvalue problem, the perturbation series about $\epsilon=0$ has a zero radius of convergence and hence is essentially useless for obtaining quantitative information for nonzero values of $\epsilon$. Hybrid solutions based on just a few terms in this series, however, provide a sequence of approximations which apparently converge to the correct solution for any $\epsilon$ as the number of terms used increases.

In addition, we have also found the hybrid method to be useful when dealing with a perturbation series with poor convergence properties within its radius of convergence. This was illustrated by Geer and Andersen (1989a) for a series which arises in the computation of the electrostatic potential field about a slender body of revolution. Here the perturbation solution is a series which contains an infinite number of terms, each one of which is an infinite series involving inverse powers of $\log \epsilon$. These series appear to converge for small values of $\epsilon$ of interest, but the rate of convergence is very slow. We were able to obtain a very meaningful approximation to the solution for these values of $\epsilon$ by using just a few of the perturbation coordinate functions in the hybrid solution.

In general, the amplitudes $\delta_{j}$ which appear in the hybrid solution need to be determined numerically. The existence of relatively simple analytical expressions for the $\delta_{j}$, such as obtained in section 3 are definitely atypical. However, the availability of such expressions for a few simple examples provides us with useful insight into the method.

As was pointed out in section 3 , the $\delta_{j}$ for our simple example have real singularities (as functions of $\epsilon$ ) which approximate some of the singularities of the actual solution. (It is often these singularities which limit the convergence (and hence the range of usefulness) of perturbation solutions.) This property of the $\delta_{j}$ seems to be a key reason why the hybrid solution can approximate functions (i.e., solutions to particular problems) which have various types of singularities. The solution of the simple example discussed by Geer and Andersen (1989a) has singularities corresponding to purely imaginary values of $\epsilon$. In this case, the corresponding $\delta_{j}$ also have purely imaginary singularities, which again approximate some of the singularities of the solution.

For the example discussed in section 4 , it was relatively straightforward to obtain two 
perturbation approximations to the solution - one valid formally for small values of $\epsilon$ and the other valid formally for large values of $\epsilon$. (This property is also illustrated for several other classes of two-point boundary value problems for ordinary differential equations in Geer and Andersen (1989b).) In any particular problem, only one of these solutions may be physically relevant. However, if they can both be constructed, it is our recommendation that this be done (although, in practice, it may be much easier to compute higher-order terms in one expansion than in the other). The reason for this is that, in general, it has been our experience that it is better to use at least one term from each of these expansions in the hybrid approximation, than to use several terms from just one of the expansions (see especially the eigenvalue example discussed by Geer and Andersen (1989b).)

It is also worth noting that only relatively simple mathematical operations are required to implement the hybrid method. In particular, once the perturbation coordinate functions have been determined, the Galerkin conditions (4) can be obtained by using a simple quadrature formula, such as Simpson's rule. Once these equations are determined, they can be solved (if necessary) by Newton's method. In practical terms, the Jacobian matrix required for this method can be well approximated by using a simple central difference formula and two more numerical quadratures for each component.

Finally, we comment that if the differential equation to be solved has no parameter, then one should be invented. The given equation should be rewritten as an equation with a parameter. For one value of the parameter the new equation should coincide with the given equation. For another value of the parameter, the new equation should reduce to an equation which somehow is simpler than the original equation, perhaps by virtue of being linear instead of nonlinear, or by having higher symmetry. Then the asymptotic expansion is performed about this simpler equation.

\section{EXTENSIONS}

Numerous variations on the hybrid technique are possible. Some, such as expansions in two or more parameters simultaneously through the use of higher-order mixed partial derivatives have already found application. Other variations of the hybrid method need further investigation. For instance, the Bubnov-Galerkin step uses the perturbation coordinate functions as both trial and test functions. Perhaps, for some equations or systems of equations, a different weighted residual technique should be used in which the set of test functions should be different from the set of trial functions. It is known, however, that for a wide variety of problems the Bubnov-Galerkin step is simple in concept, effective, and easy to implement.

The hybrid technique is effective for numerical problems as well as for problems which may be analyzed in terms of analytic functions. Many problems are far too complex because of their geometry, discontinuities or other reasons for effective analysis in terms of simple analytic functions. For these problems finite element, finite difference, RayleighRitz or other discretization techniques are commonly employed. However, the generation of series expansions and the formulation of the Galerkin step for discretized problems have direct numerical counterparts to the analytic steps employed in the study presented here. For discretized problems basis vectors in a large but finite-dimensional space take the place of the test and trial functions. The trick is to pick a small number of appropriate basis vectors to use as input to the Galerkin step, hence the term "reduced-basis method". 
We are currently investigating several possible application areas for the hybrid method and many of our preliminary results have been encouraging. In particular, we are investigating the application of the method to several eigenvalue problems, both for ordinary and partial differential equations. In addition to the quantum mechanical eigenvalue problem discussed in Andersen and Geer (1988) and Geer and Andersen (1989b) we are investigating some eigenvalue problems for partial differential equations where the boundary of the spacial domain for the problem is a perturbation of a "simple" domain for which the eigenvalue problem can be solved in closed form.

We also feel that the method is potentially very useful for solving a variety of exterior boundary value problems for partial differential equations. In particular, we have already applied the method to some model problems in slender body theory (Geer and Andersen (1989a)) with promising results, and are currently working on combining the method with some simple homotopy ideas to further increase the accuracy of slender body approximations.

The field of free and forced nonlinear vibrations is another application area we are investigating. In this area we have found the method to be particularly effective for computing resonant frequencies of certain nonlinear oscillators (Geer and Andersen (1989b)), and are also currently using the method in conjunction with the multiple time scales method to compute frequency response curves for certain forced nonlinear oscillation problems.

\section{REFERENCES}

Andersen, C M, and Geer, J F, "Power series expansions for the frequency and period of the limit cycle of the van der Pol equation", SIAM J Appl Math 42 (1982), 678-693.

Andersen, C M, and Geer, J F, "Investigating a hybrid perturbation Galerkin technique using computer algebra", NASA Langley Research Center ICASE Report No. 88-65, 1988.

DiPrima, R C, "Higher-order approximations in the asymptotic solution of the Reynolds equation for slider bearings at high bearing numbers", $J$ Lub Tech, 91, (1969), 45-51.

Fink, J P, and Rheinbolt, W C, "On the error behavior of the reduced basis technique for nonlinear finite element approximations", $Z A M M 63$ (1983), 21-28.

Galerkin, B G, Vestnik Inzhenerov i Tekhnikovi, Tech. 19 (1915), 897-908.

Geer, J F, and Andersen, C M, "A hybrid perturbation Galerkin technique with applications to slender body theory", SIAM J Appl Math 49, (1989) 344-361.

Geer, J F, and Andersen, C M, "A hybrid perturbation Galerkin method which combines multiple expansions", NASA Langley Research Center ICASE Report No. 89-8, 1989.

Nayfeh, A, Perturbation Methods, Wiley (New York), 1973.

Noor, A K, "Recent advances in reduction problems for nonlinear problems", Computers and Structures 13 (1981), 31-44.

Noor, A K, "Hybrid analytical technique for nonlinear analysis of structures", AIA $J \mathbf{2 3}$ (1985), 938-946.

Noor, A K, Andersen, C M, and Peters, J M, "Global-local approach for nonlinear shell analysis", Proc. 7th ASCE Conf. on Electronic Comp., Washington U., St. Louis, Mo., Aug. 1979, 634-657.

Noor, A K, Andersen, C M, and Peters, J M, "Reduced basis technique for collapse analysis of shells", A IA A J, 19 (1981), 393-397. 
Noor, A K, Andersen, C M, and Tanner, J, "Nonlinear analysis of shells of revolution via semi-analytic finite elements with applications to tires", Computational Mechanics '88, ed. by S. N. Atluri and G. Yagawa, Springer-Verlag, Vol. 1, 1988, 27.i.1-27.i.4.

Noor, A K, Balch, C D, and Shibut, M A, "Reduction methods for nonlinear steady-state thermal analysis", Int J Num Meth Eng 20 (1984), 1323-1348.

Noor, A K, and Balch, C D, "Hybrid perturbation/Bubnov-Galerkin technique for nonlinear thermal analysis", AIAA J 22 (1984), 287-294.

Noor, A K, and Peters, J M, "Bifurcation and post-buckling analysis of laminated composite plates via reduced basis technique", Comp Meth Appl Mech Eng, 29 (1981), 271-295. Noor, A K, and Peters, J M, "Mixed models and reduced/selective integration displacement models for nonlinear analysis of curved beams", Int J Num Meth Eng 17 (1981), 615-631.

Noor, A K, and Peters, J M, "Multiple-parameter reduced basis technique for bifurcation and post-buckling analysis of composite plates", Int J Num Meth Eng 19 (1983), 17831803.

Noor, A K, and Peters, J M, "Nonlinear analysis via global-local mixed finite element approach", Int $J$ Num Meth Eng 15 (1980), 1363-1380.

Noor, A K, and Peters, J M, "Recent advances in reduction methods for instability analysis of structures", Computers and Structures 16 (1983), 67-80.

Noor, A K, Peters, J M, and Andersen, C M, "Mixed models and reduction techniques for large-rotation nonlinear problems", Comp Meth Appl Mech Eng 44 (1984), 67-89.

Van Dyke, M, "Analysis and Improvement of perturbation series", Quart J Mech Appl Math 27 (1974), 423-450.

Van Dyke, M, Perturbation methods in fluid mechanics, Parabolic Press (Stanford), 1975.

Wolfram, S, Mathematica - a system for doing mathematics by computer, Addison-Wesley (Redwood City, CA), 1988. 


\section{LIST OF FIGURES}

Figure 1. Exact solution for simple differential equation of Section 3. $u(x ; \epsilon)$ as a funtion of $x$ and $\omega=\sqrt{(\epsilon)}$

Figure 2. Comparison for the simple equation (5) of exact solutions (6), perturbation solutions (7) with $N=2$ and $N=4$, and hybrid solutions (8) with $N=2$. The parameter $\epsilon$ has values 2,4 , and 6 .

Figure 3. Comparison for the simple equation (5) of exact solutions (6), perturbation solutions (7) with $N=2$ and $N=4$, and hybrid solutions (8) with $N=2$. The parameter $\epsilon$ has values $(0.9 \pi)^{2},(0.97 \pi)^{2}$, and $(1.03 \pi)^{2}$, and $(1.1 \pi)^{2}$. The perturbation solutions are indistinguishable from one another.

Figure 4. Comparison for the simple equation (5) of exact solutions (6) and hybrid solutions (8) with $N=2$ and $N=4$. The parameter $\epsilon$ has the value $(2 . \pi)^{2}$.

Figure 5. Comparison for the simple equation (5) of exact solutions (6) and hybrid solutions (8) with $N=2$ and $N=4$. The parameter $\epsilon$ has the value $(3.05 \pi)^{2}$.

Figure 6. Comparison of solutions of the wedge-shaped slider bearing equation (13). One-term small- $\epsilon$ perturbation solution (14), one-term large- $\epsilon$ perturbation solution (16), two-term hybrid solution (19), and shooting-method (numerical) solution.

Figure 7. Comparison of pressure distributions for a wedge-shaped bearing with $\epsilon=$ $0.1,0.2,0.3$ and 1.0 as computed by hybrid and numerical methods.

Figure 8. The amplitudes, $\delta_{1}$ (small- $\epsilon$ term) and $\delta_{2}$ (large- $\epsilon$ term), in (19) for $0<\epsilon<2$.

Figure 9. Comparison of pressure distributions for a bearing with a parabolic film thickness as computed by hybrid, perturbation and numerical methods.

Figure 10. Comparison of pressure distributions for a bearing with a cubic film thickness as computed by hybrid, perturbation and numerical methods.

Figure 11. Comparison of load carrying capacity calculations for the wedge-shaped bearing as computed by two-term hybrid, one-term small- $\epsilon$ perturbation, one-term large- $\epsilon$ perturbation, and direct numerical methods. 

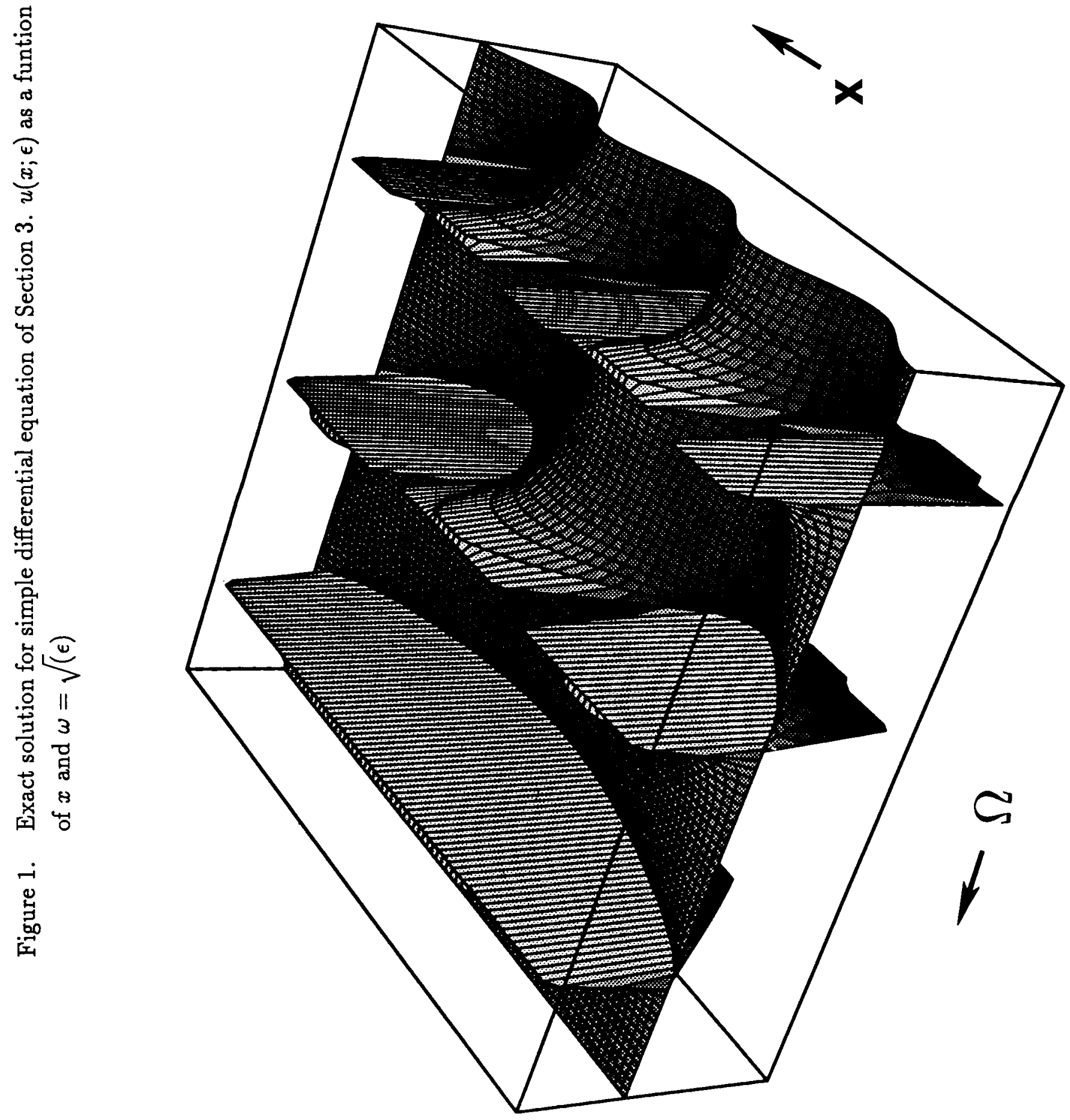

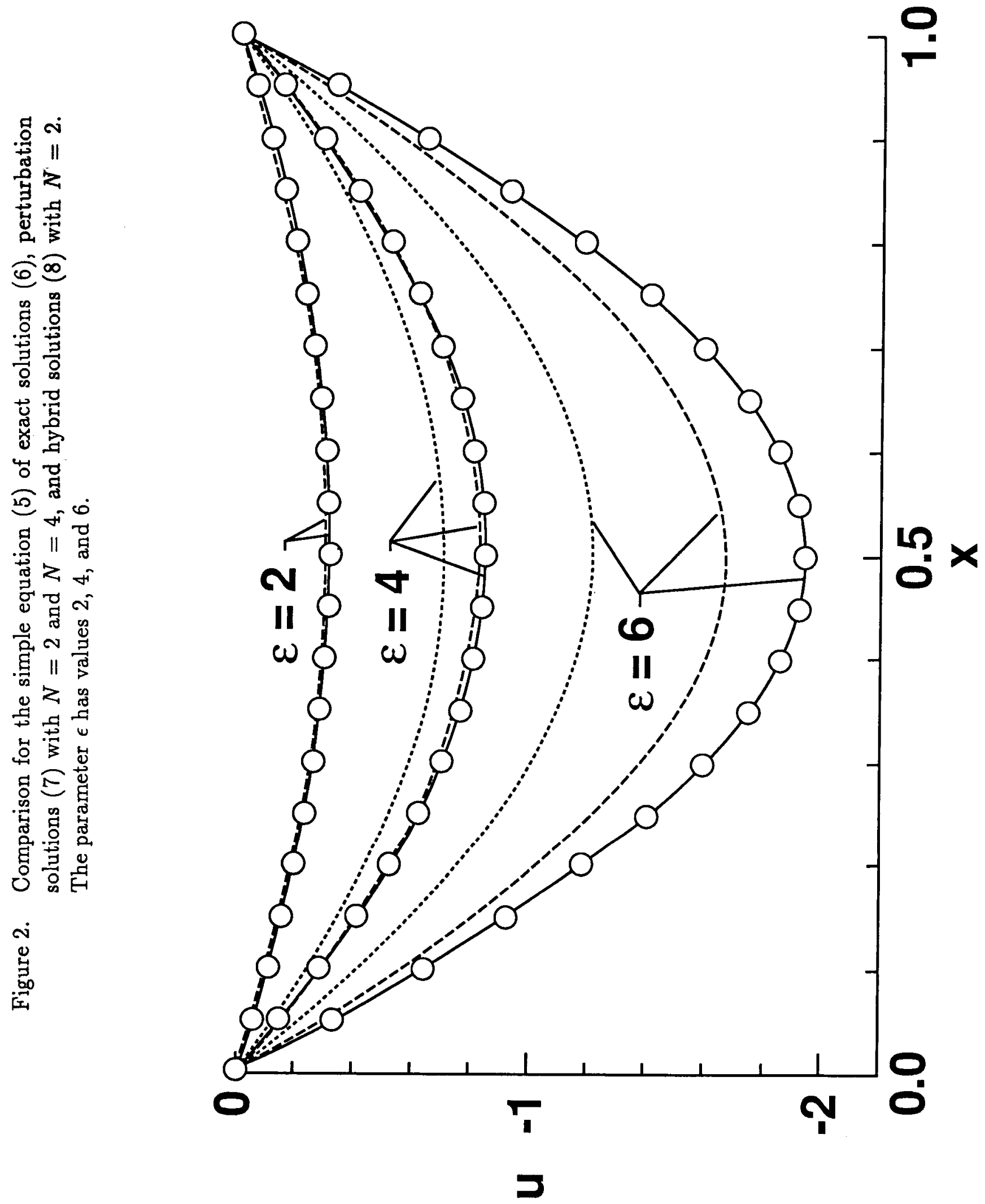


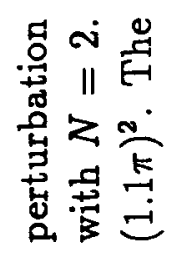

क요

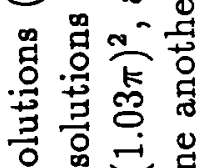

흐웅

苞热

(3) 牙

栉 $\frac{0}{0}$

(1) क व

व

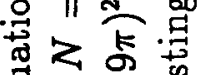

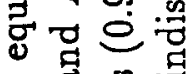

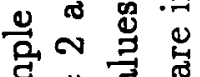

द्g 11

之

台率出

송ㅁㅇ

骂品营売

\%

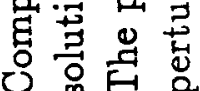

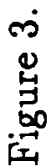

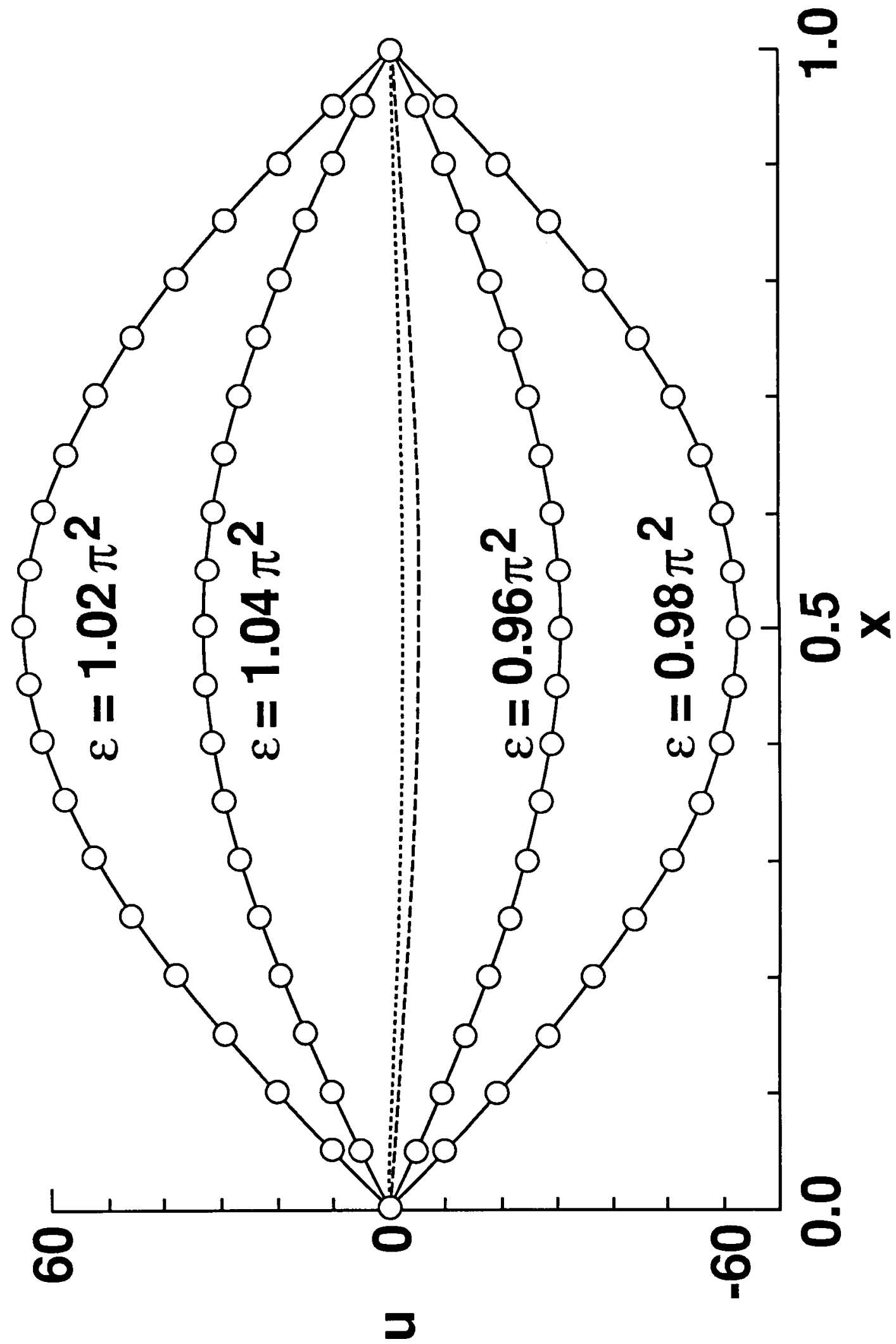



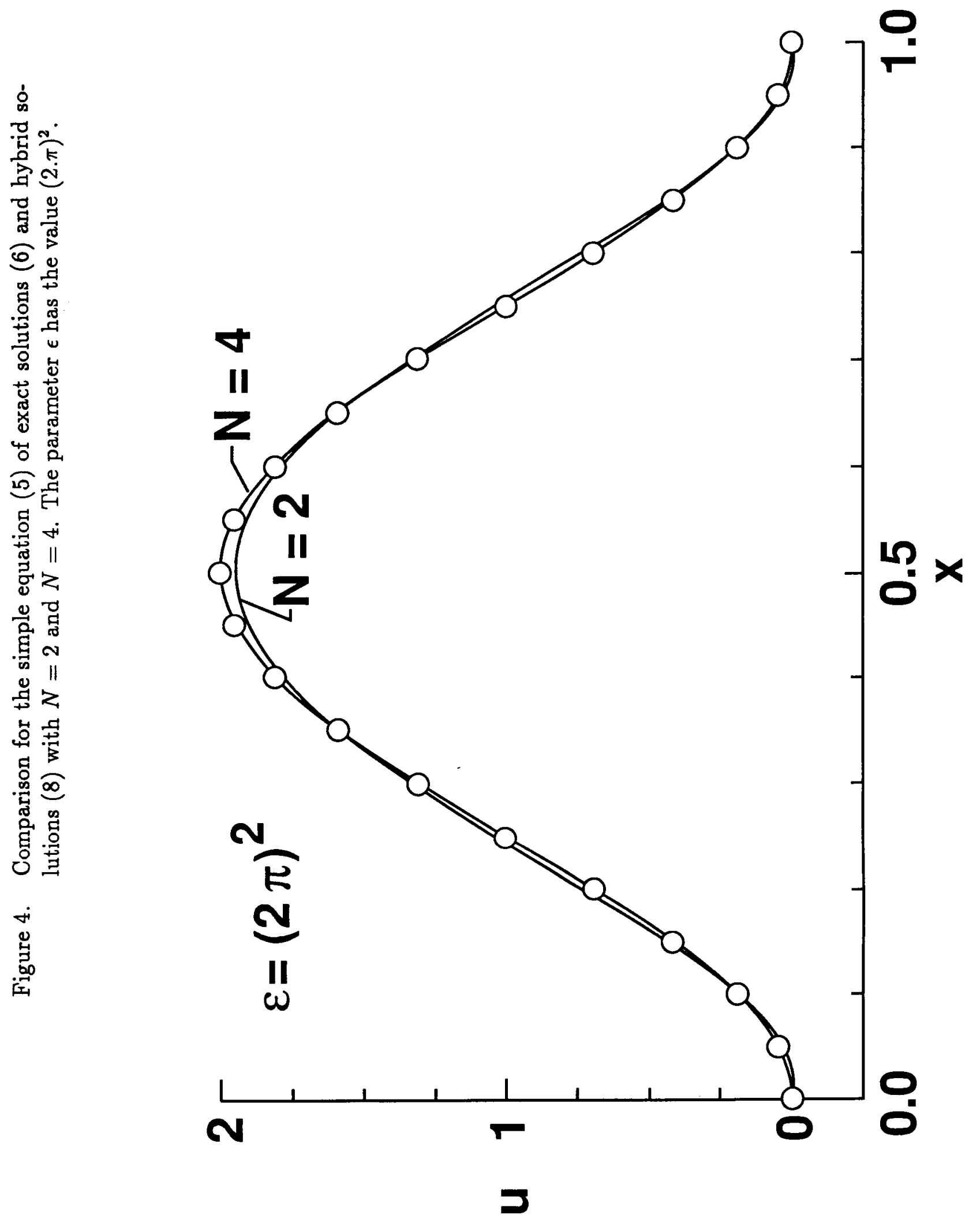

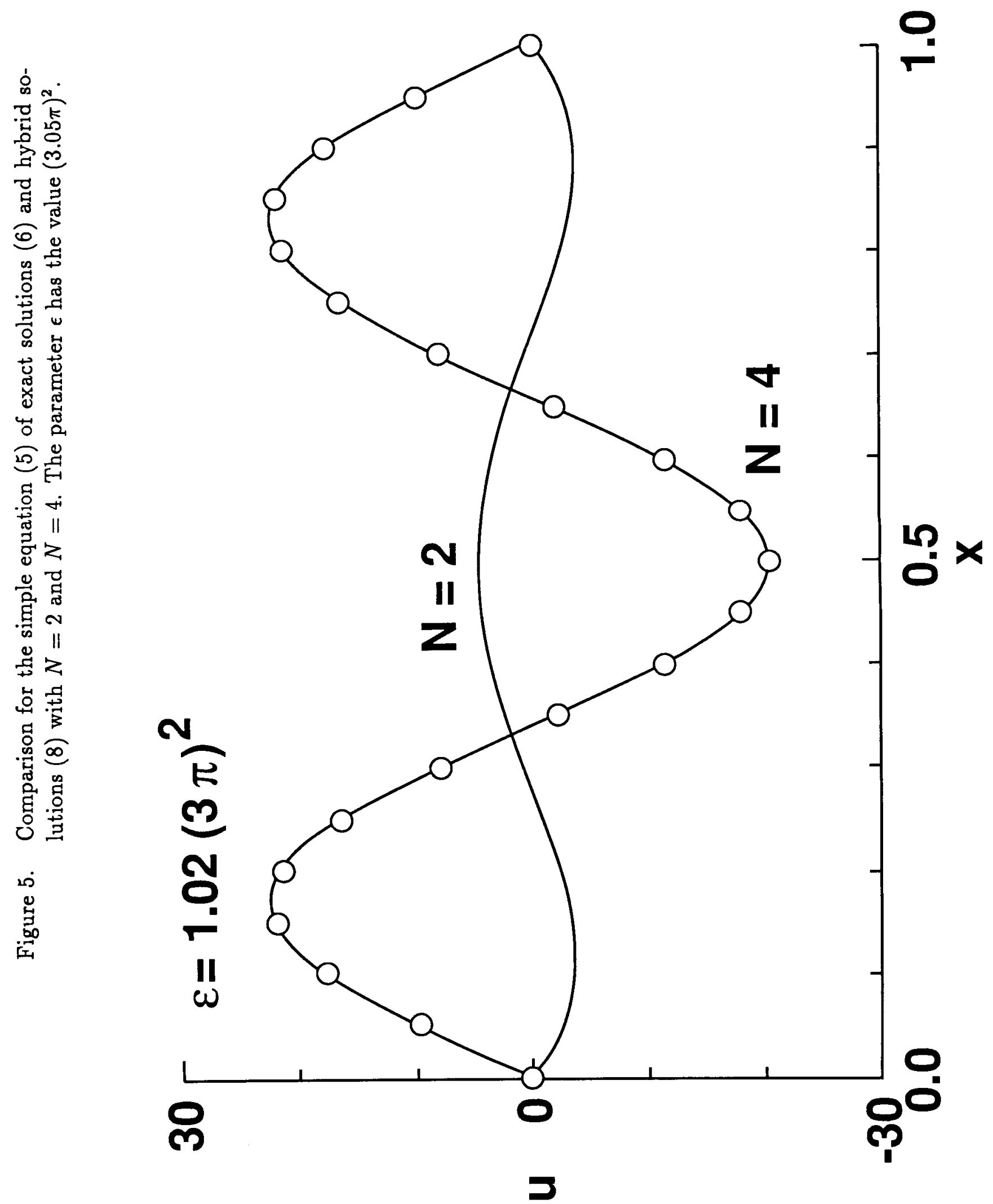

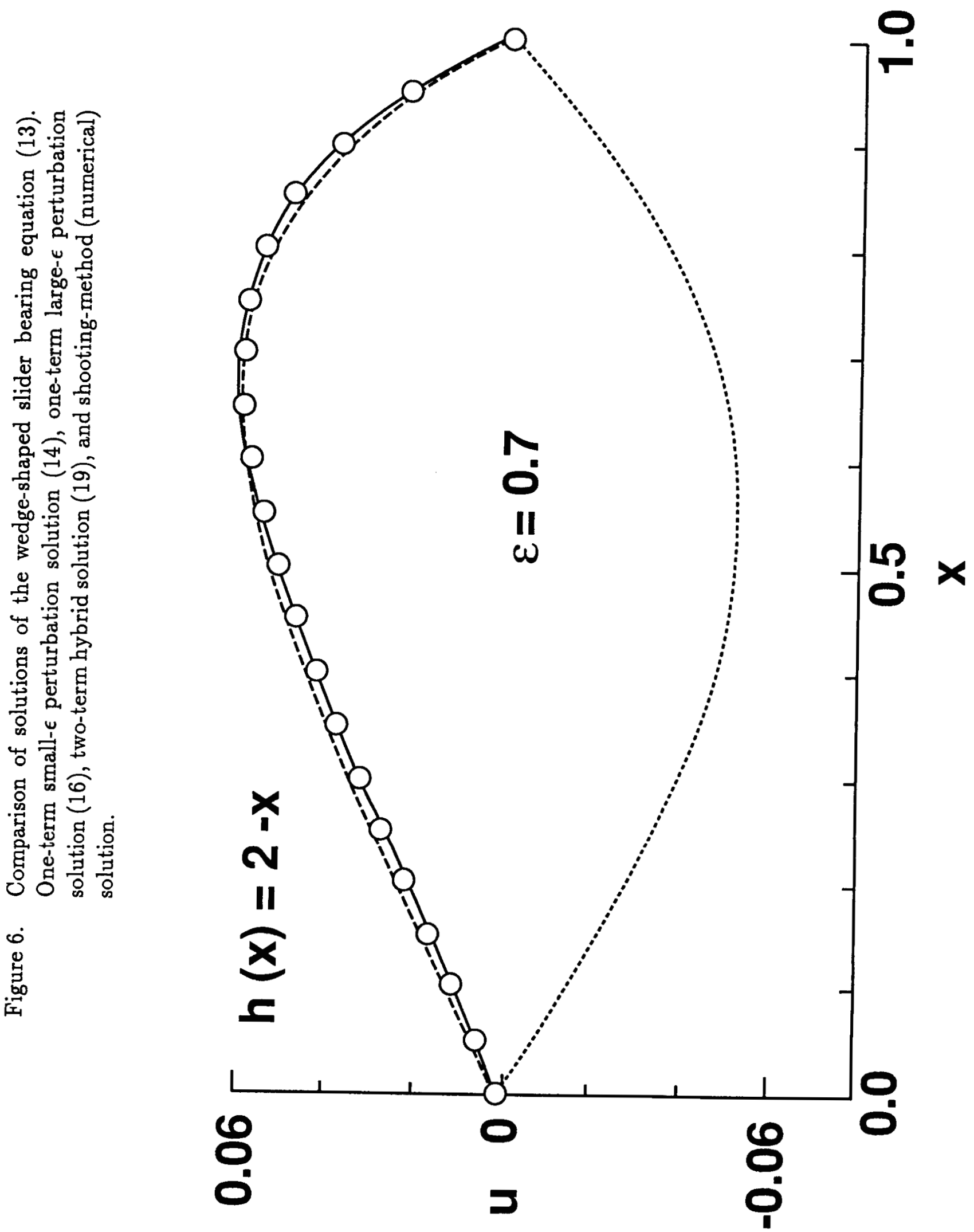

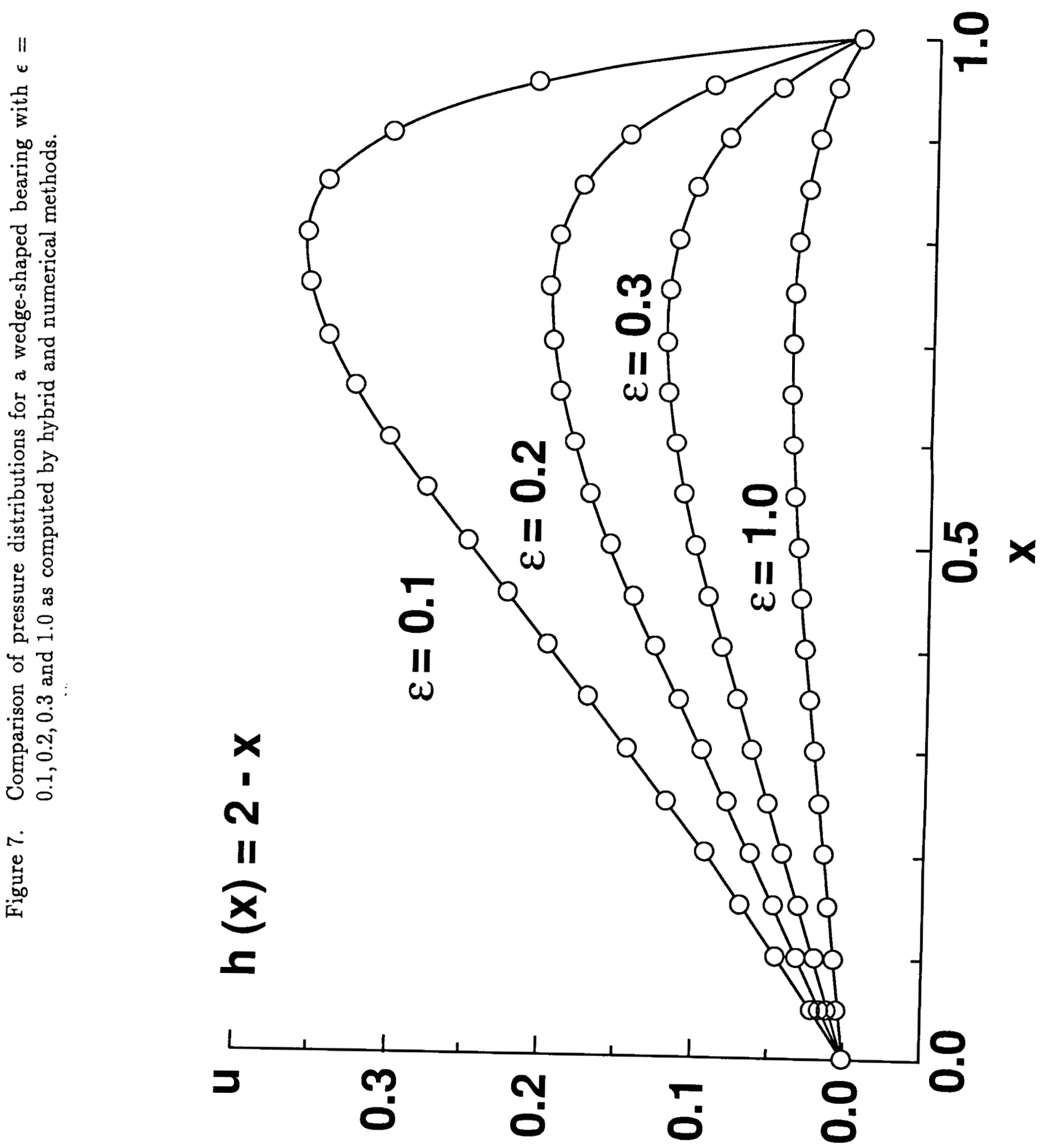


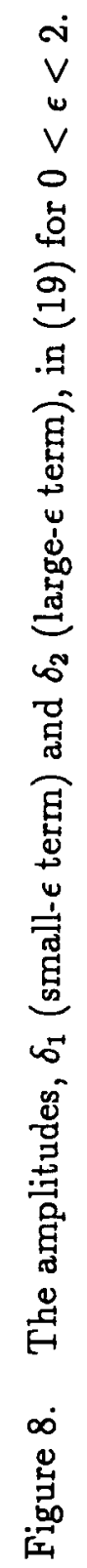

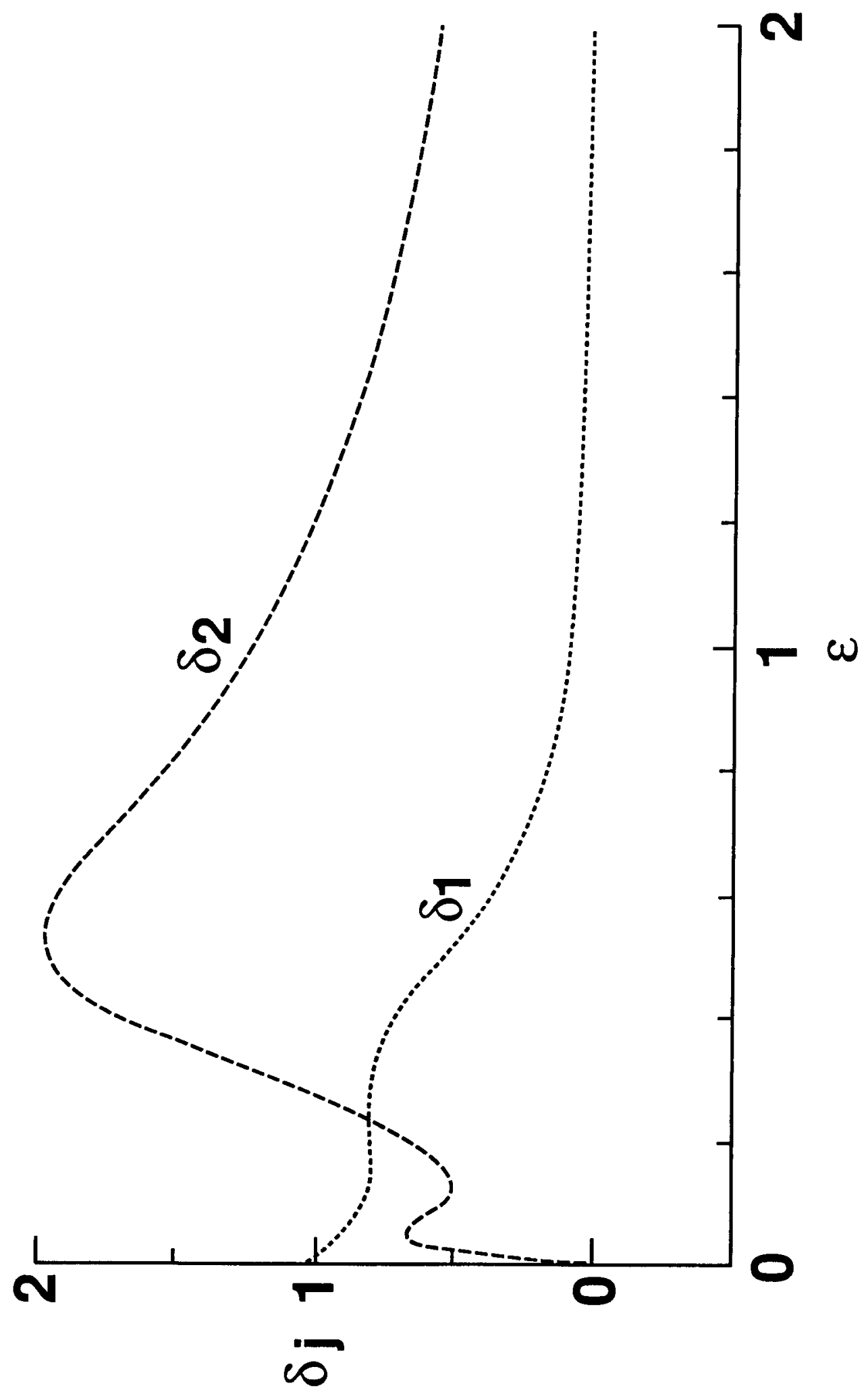



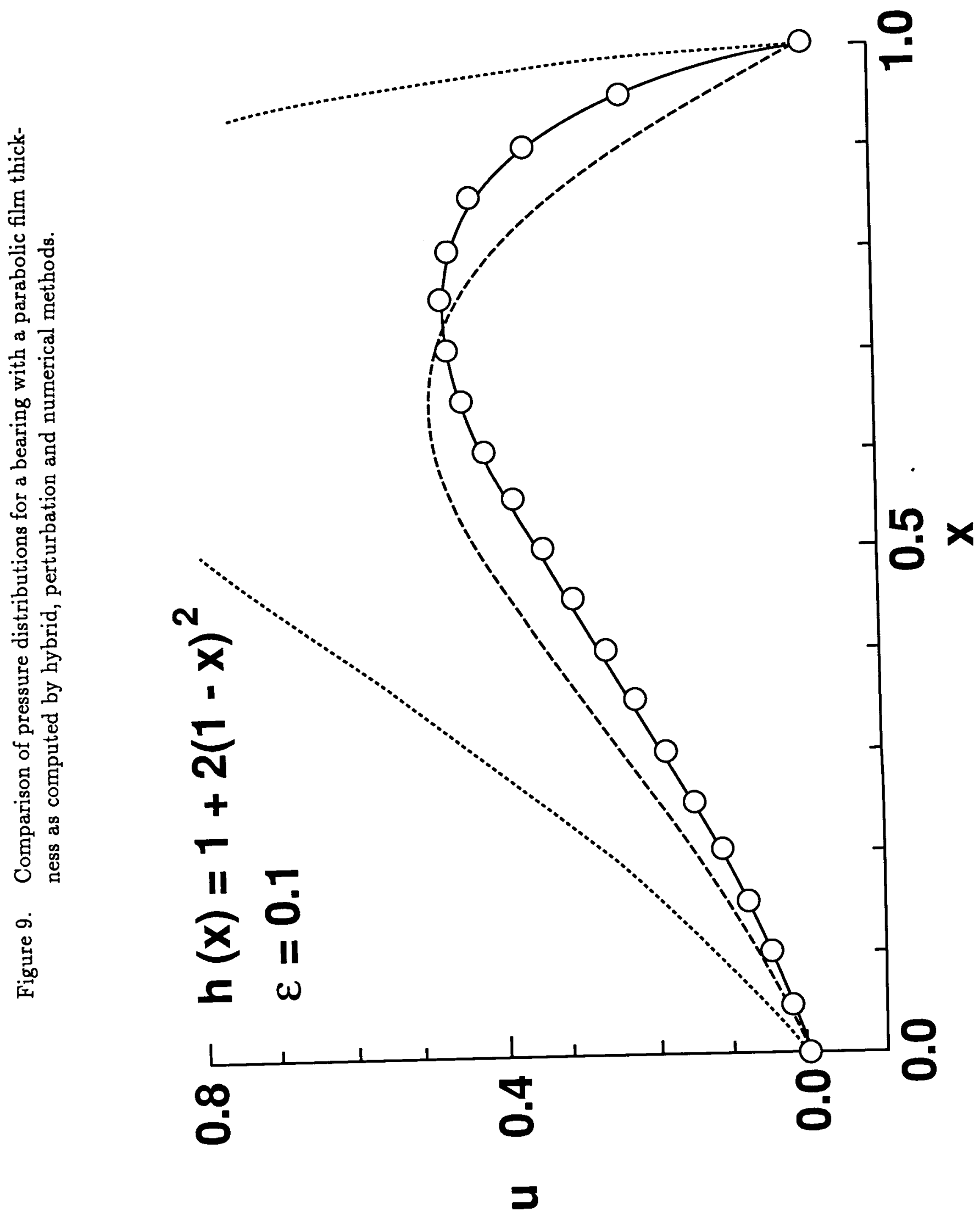

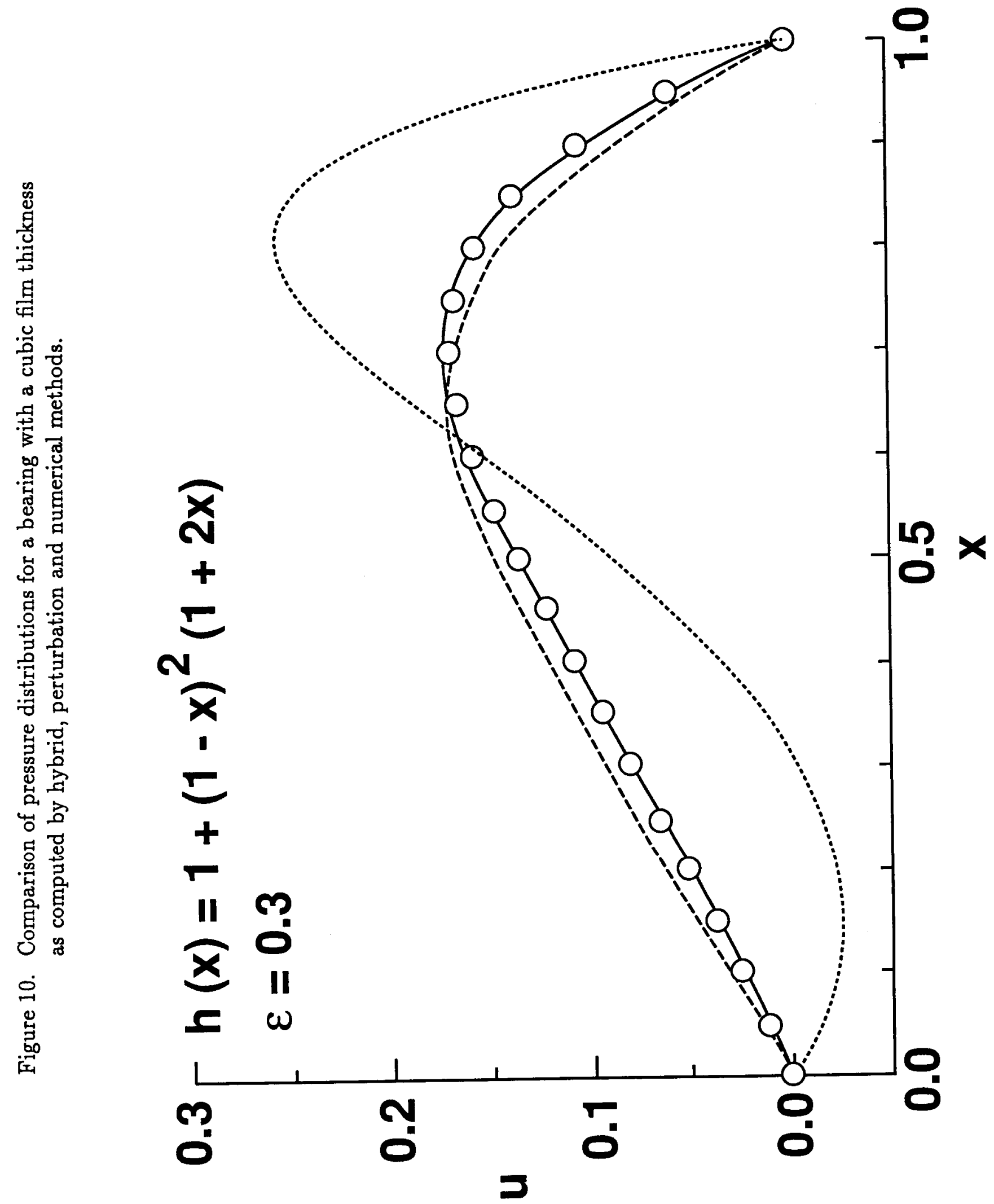

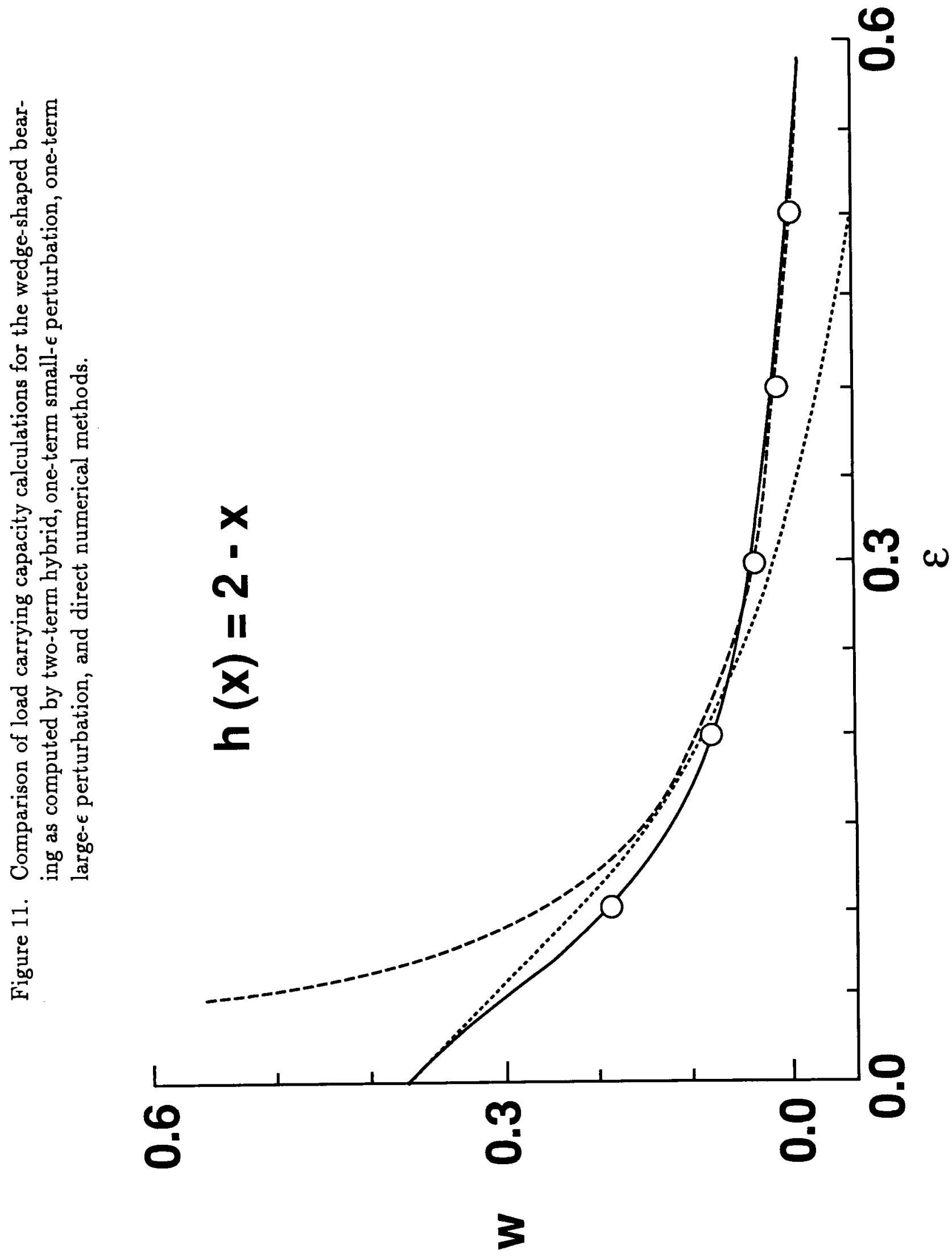


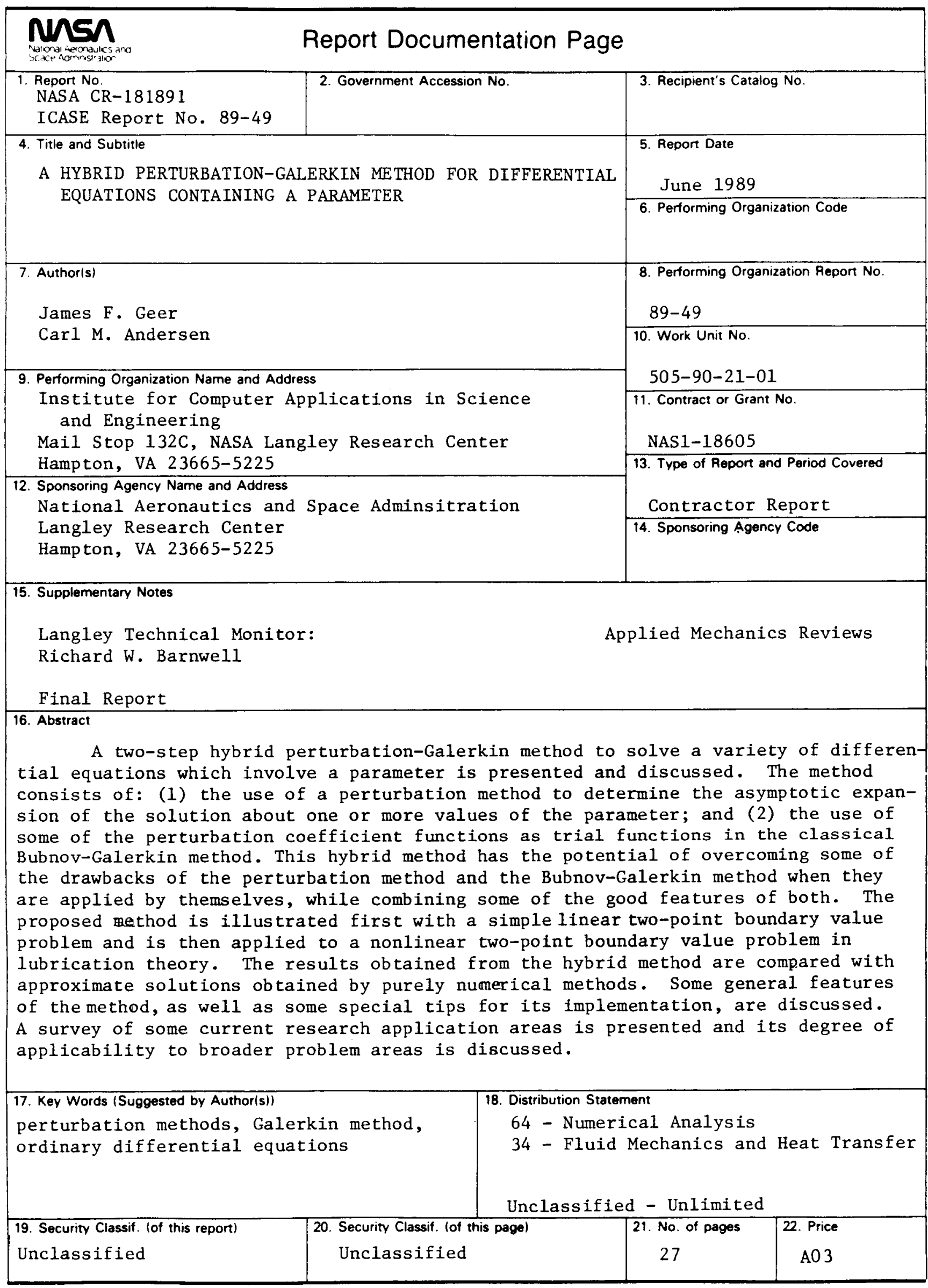

